\title{
AVALIAÇÃO COMPARATIVA DA COMPOSIÇÃO QUÍMICA DE DROGAS VEGETAIS E EXTRATOS DE Calendula officinalis L. (Asteraceae) CULTIVADA COM VARIAÇÃO NA ADUBAÇÃO E NA COBERTURA DE SOLO
}

\author{
COMPARATIVE EVALUATION OF CHEMICAL COMPOSITION OF PLANT \\ EXTRACTS AND DRUGS OF Calendula officinalis L. (Asteraceae) CULTIVATED \\ WITH VARIATION OF FERTILIZATION AND MULCHING
}

\author{
Júlio Cézar BORELLA ${ }^{1}$; Andreia MARINCEK²; Marissa Ferreira RODRIGUES ${ }^{2}$
}

\begin{abstract}
'Docente orientador do Curso de Ciências Farmacêuticas. Universidade de Ribeirão Preto - UNAERP, Disciplina de Farmacognosia. E-mail para correspondência:jborella@unaerp.br

${ }^{2}$ Acadêmica do Curso de Ciências Farmacêuticas. Universidade de Ribeirão Preto - UNAERP,

Disciplina de Farmacognosia.
\end{abstract}

\section{RESUMO:}

O presente trabalho tem por objetivo avaliar alguns dos constituintes químicos da composição de insumos fitoterápicos (drogas vegetais, tinturas e extratos glicólicos) de Calendula officinalis $L$. que foi cultivada com diferentes tipos de adubação e cobertura de solo. O experimento realizado para o cultivo da espécie medicinal foi constituído de seis tratamentos, sendo 1. adubação química (100 kg de $\mathrm{N} \mathrm{ha}^{-1}+50 \mathrm{~kg}$ de $\mathrm{P}$ ha $\left.{ }^{-1}\right) ; 2$. adubação química + cobertura (grama seca); 3. adubação orgânica (esterco bovino curtido $50 \mathrm{t} \mathrm{ha}^{-1}$ ); 4. adubação orgânica + cobertura; 5. sem adubação química ou orgânica + cobertura; 6. sem adubação química ou orgânica e sem cobertura. As inflorescências foram colhidas, secas e moídas obtendo-se as drogas vegetais. Com elas foram produzidos extratos utilizando-se como solventes I. etanol:água 62:38 (v/v) e II. propilenoglicol:água 90:10 (v/v). Com as drogas vegetais foi calculado o índice de espuma (análise semi-quantitativa para saponinas). Houve diferenças significativas entre a droga cultivada com adubação orgânica sem cobertura morta (IE =198) e os demais tratamentos (IE entre 100-181). Com os extratos foi realizado screening fitoquímico para evidenciação das principais categorias de constituintes e mostrou que o tipo de cultivo realizado e o solvente utilizado na extração não influenciaram a composição química no aspecto qualitativo. Foram evidenciados nos extratos hidroetanólicos (tinturas) e nos extratos hidroglicólicos os compostos fenólicos (taninos catequínicos), flavonoides, saponinas, alcaloides e cumarinas.

Palavras-chave: Plantas Medicinais, Cultivo, Controle de qualidade, Extratos vegetais.

\section{ABSTRACT:}

This study aims to evaluate some of the chemical constituents in the composition of herbal ingredients (plant drugs, tinctures and glycolic extracts) Calendula officinalis L. (Marigold) which was cultivated with different types of fertilizer and mulch. The cultivation experiment was constituted of six treatments: 1 . chemical fertilization $(100 \mathrm{~kg}$ $\mathrm{N} \mathrm{ha}^{-1}+50 \mathrm{~kg} \mathrm{P} \mathrm{ha}^{-1}$ ); 2. chemical fertilization + mulch (dry grass); 3. organic fertilization (cured bovine manure 50 t ha $^{-1}$ ); 4. organic fertilization + mulch; 5. no chemical or organic fertilization + mulch; 6. no chemical or organic fertilization and no mulch. The 
inflorescences were harvested, dried and ground. Extractions were performed with plant drugs using as solvent: I. ethanol: water 62:38 (v/v) and II. propylene glycol: water 90:10 (v/v). With plant drugs were obtaining the foam index (semi-quantitative analysis for saponin). There were significant differences between plant drugs those grown with organic fertilizer without mulching $(\mathrm{FI}=198)$ and the other treatments ( $\mathrm{Fl}$ between 100181). Phytochemical screening was carried out with the extracts to show the main categories of constituents. The result showed that the type of cultivation performed and the solvent used did not affect the chemical composition of the qualitative aspect. Phenolics compounds (condensed tannins), flavonoids, saponins, alkaloids and coumarins were detected in hydroalcoholic extracts (tinctures) and hydro-glycolic extracts.

Keywords: Medicinal plants, Cultivation, Quality Control, Plant Extracts.

\section{INTRODUÇÃO}

Espécies vegetais com utilização terapêutica, muitas vezes são conceituadas como verdadeiros laboratórios de síntese de princípios ativos (CRAGG; NEWMAN, 2009). No entanto, diferentemente disso, elas são seres vivos e podem sofrer ao longo de sua vida, influências genéticas e ambientais, podendo resultar em alterações na sua composição química, principalmente relacionadas aos metabólitos secundários, alterando também suas propriedades farmacológicas (GOTTLIEB; BORIN, 2012).

Alguns fatores ambientais podem acarretar essas modificações como as condições do solo e disponibilidade de nutrientes (GOBBO-NETO; LOPES, 2007). Condições adequadas de adubação e proteção do solo podem, em alguns casos, melhorar a disponibilização de nutrientes para o vegetal, resultando em melhor desenvolvimento da planta e de seu metabolismo. Outro fator que também interfere na composição química das preparações à base de espécies vegetais, inclusive de fitoterápicos, é o processo de extração da planta. Existem vários fatores que podem interferir nesse momento, entre eles, o líquido extrator utilizado. Ele é escolhido em função do tipo de princípio ativo que se deseja extrair, optando por solventes de menor ou maior polaridade. De forma geral, os últimos têm menor seletividade e por isso, são rotineiramente empregados nas preparações quando se tenta extrair da planta, a integridade de seu fitocomplexo, importante para a eficácia do fitoterápico (SONAGLIO et al., 2007).

Calendula officinalis L., pertencente à família Asteraceae, é uma espécie utilizada terapeuticamente por suas ações antiinflamatórias e cicatrizantes e sua principal forma de utilização é em preparações tópicas (LEACH, 2008). Adroga vegetal derivada dessa planta são as inflorescências secas, separadas do receptáculo e das brácteas involucrais (FARMACOPEIA BRASILEIRA, 2010). Essa espécie possui metabólitos que estão relacionados com suas atividades farmacológicas e são citados na literatura os flavonoides, saponinas, cumarinas, ácidos fenólicos, polissacarídeos de alto peso molecular e terpenoides como óleos voláteis e carotenoides (CITADINI- 
ZANETTE et al., 2012; KHALID; TEIXEIRADA SILVA, 2012; FERNANDES et al., 2013).

É frequente a utilização de tinturas e extratos glicólicos na produção de formulações fitoterápicas contendo Calêndula e é também rotineiro a substituição de um extrato pelo outro, objetivando dar mais estabilidade a essas preparações. Extratos hidroetanólicos e extratos hidroglicólicos, contendo propilenoglicol, são os mais utilizados na composição dessas preparações (NUNES et al., 2009; FORMULÁRIO DE FITOTERÁPICOS, 2010; BORELLA et al. 2010; BORELLA et al. 2011; BORELLAet al. 2012; BORELLA; TEIXEIRA2013).

Deste modo, torna-se necessária avaliação da composição química dos insumos utilizados nas preparações fitoterápicas para manutenção de sua eficácia e segurança de uso. Atualmente há a disposição grande variedade de técnicas analíticas para essa finalidade. O screening fitoquímico é uma conduta na qual, através de vários testes, embasados nas características físicas e químicas dos compostos, se conclui a respeito da presença deles nos extratos vegetais (BARBOSA et al., 2004). Também podem ser citadas para essa finalidade, as técnicas tipicamente farmacognósticas, como o índice de espuma, análise semi-quantitativa de saponinas que pode ser empregada para comparação de drogas vegetais, já que nesse ensaio se estabelece uma relação entre a quantidade de espuma produzida e as saponinas espumídicas presentes (FARMACOPEIABRASILEIRA, 2010).

Sendo assim, o presente trabalho apresenta estudo desenvolvido sobre a droga vegetal de $C$. officinalis obtida com diferentes condições de cultivo e sobre seus extratos hidroetanólicos (tinturas) e hidroglicólicos (extratos glicólicos), no sentido de averiguar diferenças na composição química desses insumos.

\section{MATERIALE MÉTODOS}

\subsection{Obtenção da droga vegetal}

O experimento relativo ao cultivo da espécie medicinal foi realizado em latitude $21^{\circ} 10^{\prime} 42$ "S, longitude 47039'33"O, altitude de 849 metros e se caracteriza por possuir solo de fertilidade média, topografia não acidentada, clima tropical úmido, com estação chuvosa no verão e seca no inverno. Exemplares do material cultivado foram herborizados e depositados no Herbário de Plantas Medicinais do Laboratório de Biotecnologia da Universidade de Ribeirão Preto, recebendo o número de catalogação HPMU 1326. C. officinalis foi cultivada a partir das sementes (variedade Bonina - Isla Pak) e a germinação foi feita em bandejas plásticas contendo terra, composto orgânico e areia, na proporção de 1:1:1. Elas foram protegidas do sol e irrigadas diariamente. Após duas semanas foi realizado desbaste e com um mês as mudas foram levadas a campo, para transplante no local definitivo (ao sol pleno). O solo foi previamente 
analisado utilizando uma amostra de profundidade de $20 \mathrm{~cm}$, tendo como resultados para macronutrientes: $\mathrm{pH}=4,9 ; \mathrm{P}=45 \mathrm{mg} \mathrm{dm}{ }^{-3} ; \mathrm{Ca}=21 \mathrm{mmol}_{\mathrm{c}} \mathrm{dm}^{-3} ; \mathrm{Mg}^{-3}=6 \mathrm{mmol}_{\mathrm{c}} \mathrm{dm}^{-3}$; $\mathrm{K}=1,4 \mathrm{mmol}_{\mathrm{c}} \mathrm{dm}^{-3} ; \mathrm{Al}=0 \mathrm{mmol}_{\mathrm{c}} \mathrm{dm}^{-3} ; \mathrm{H}+\mathrm{Al}=52 \mathrm{mmol}_{\mathrm{c}} \mathrm{dm}^{-3} ;$ matéria orgânica $=32 \mathrm{~g} \mathrm{dm}^{-}$ 3; $\mathrm{V}=35 \%$; micronutrientes: $\mathrm{B}=0,23 \mathrm{mg} \mathrm{dm}^{-3} ; \mathrm{Cu}=4,2 \mathrm{mg} \mathrm{dm}^{-3} ; \mathrm{Fe}=29 \mathrm{mg} \mathrm{dm}^{-3} ; \mathrm{Mn}=$ $6,1 \mathrm{mg} \mathrm{dm}{ }^{-3} ; \mathrm{Zn}=1,5 \mathrm{mg} \mathrm{dm}^{-3} ;$ Granulometria: Areia total $=217 \mathrm{~g} \mathrm{~kg}^{-1} ;$ Areia grossa $=$ $140 \mathrm{~g} \mathrm{~kg}^{-1}$; Areia fina $=77 \mathrm{~g} \mathrm{~kg}^{-1}$ Silte $=600 \mathrm{~g} \mathrm{~kg}^{-1} ;$ Argila $=184 \mathrm{~g} \mathrm{~kg}^{-1}$. Procedimentos de calagem e elevação de saturação de bases a $50 \%$ foram realizados para melhorar a disponibilidade dos nutrientes para absorção do vegetal. As mudas foram transplantadas em 18 parcelas, com $6 \mathrm{~m}^{2}$ cada uma, contendo 25 plantas por parcela $\left(4,2\right.$ plantas $\left.\mathrm{m}^{-2}\right)$. Os tratamentos foram realizados em triplicada e tiveram as seguintes características:

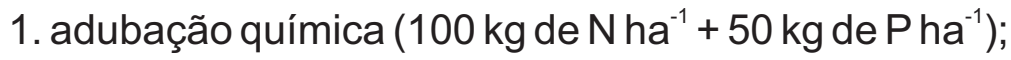

2. adubação química ( $100 \mathrm{~kg}$ de $\mathrm{N} \mathrm{ha}^{-1}+50 \mathrm{~kg}$ de $\mathrm{P}$ ha $\left.{ }^{-1}\right)+$ cobertura morta de grama seca;

3. adubação orgânica (esterco bovino curtido 50 tha $^{-1}$ );

4. adubação orgânica (esterco bovino curtido $50 \mathrm{t} \mathrm{ha}^{-1}$ ) + cobertura morta de grama seca;

5. sem adubação química ou orgânica + cobertura morta de grama seca;

6. sem adubação química ou orgânica e sem cobertura morta.

Durante seis meses (julho a dezembro), enquanto ocorreu o desenvolvimento das plantas, a temperatura variou de 13 a $33^{\circ} \mathrm{C}$ e a precipitação pluviométrica foi de 523 $\mathrm{mm}$. Houve irrigação diária, conforme a necessidade e foi realizada a manutenção das parcelas em relação à cobertura morta e retirada de ervas daninhas. Os adubos químicos utilizados foram uréia, como fonte de nitrogênio e superfosfato simples $\left(\mathrm{P}_{2} \mathrm{O}_{5}\right)$, como fonte de fósforo. Foram aplicados de uma só vez para o fósforo (no transplante) e em duas parcelas para o nitrogênio (no transplante e após 30 dias). A adubação orgânica (esterco bovino) foi realizada uma semana antes do transplante. A colheita foi iniciada 3 meses após o transplante e terminou 6 meses depois. As inflorescências foram colhidas nesse período, em dias alternados e agrupadas por tratamento. A secagem foi realizada em estufa de ar circulante a $35^{\circ} \mathrm{C}$, em seguida a droga vegetal foi separada e moída (granulometria entre 180-355 $\mu \mathrm{m}$ ).

\subsection{Obtenção dos extratos vegetais}

Cada droga vegetal diferenciada pelo tratamento realizado em seu cultivo foi submetida a processo extrativo por percolação (FORMULÁRIO DE FITOTERÁPICOS, 2010), utilizando dois tipos de líquido extrator:

I. Etanol:Água 62:38(v/v)

II. Propilenoglicol:Água 90:10 (v/v) 
A proporção entre droga vegetal e líquido extrator utilizado para desenvolvimento do processo extrativo foi de 1:5, com velocidade de gotejamento de $1 \mathrm{~mL} / \mathrm{min}$ para cada $100 \mathrm{~g}$ de droga utilizada. Deste modo, foram obtidos doze tipos de extratos, seis hidroetanólicos (tinturas) e seis hidroglicólicos (extrato glicólico).

\subsection{Screening fitoquímico dos extratos}

Foi realizada técnica para avaliação da presença de algumas categorias de produtos naturais nos extratos hidroetanólicos e hidroglicólicos de inflorescências de $C$. officinalis. Para isso eles foram primeiramente submetidos a processo de secagem em evaporador rotativo (para os extratos hidroetanólicos) e na manta de aquecimento (para os extratos hidroglicólicos). Houve necessidade de finalizar a secagem com uso de estufa a $100{ }^{\circ} \mathrm{C}$ e a manutenção desse estado de secura foi obtido armazenando os extratos em dessecador com sílica.

Os testes foram realizados conforme descrito por Barbosa et al. (2004), com o objetivo de evidenciar compostos fenólicos - taninos (reação com solução de cloreto férrico 1\%), flavonoides (reação de Shinoda), saponinas (teste afrogênico), esteroides e triterpenos (teste de Liebermann-Burchard), alcaloides (testes com os reativos de Dragendorff, Mayer e Bouchardat), heterosídeos cardiotônicos (testes com o reativo de Kedde e com solução de nitroprussiato de sódio 5\%); carotenoides (reação com solução clorofórmica saturada de tricloreto de antimônio); cumarinas (fluorescência com irradiação UV em meio alcalino) e antraquinonas (teste de Borntraeger). Todos os testes foram realizados em triplicata.

\section{Determinação do índice de espuma das drogas vegetais}

A técnica utilizada foi a descrita pela FARMACOPEIABRASILEIRAV ed. (2010) onde se obtém $100 \mathrm{~mL}$ de extrato aquoso (decoto) a partir de $1 \mathrm{~g}$ da droga vegetal. Este sofre uma série de diluições, produzindo soluções de concentrações que variam de $10 \%(\mathrm{v} / \mathrm{v})$ até $90 \%$ (v/v). Essas soluções e o decoto original foram energicamente agitados por 15 segundos através de dinamizador Autic e deixados em repouso por 15 minutos. No final desse tempo se pesquisou na série, qual das soluções é a mais diluída e que apresentou anel de espuma com $1 \mathrm{~cm}$ de espessura. Para obtenção do índice de espuma da droga foi calculado:

$$
\mathrm{IE}=\frac{\text { volume de extrato (decoto) utilizado no tubo escolhido }}{1000}
$$

Por conceito, o índice de espuma expressa a quantidade de líquido extrator que 
seria necessário para produzir um extrato aquoso (decoto) com um grama da droga vegetal que exiba anel de espuma com $1 \mathrm{~cm}$ de espessura após agitados, nas condições do experimento.

A análise descrita foi realizada em triplicada para cada uma das drogas vegetais e os resultados foram submetidos à análise de variância (ANOVA) e posteriormente avaliados no teste de comparações múltiplas de Tukey-Kramer, ao nível de $5 \%$ de probabilidade.

\section{RESULTADOS E DISCUSSÃO}

Os resultados do screening fitoquímico dos extratos estão sumarizados na tabela 1. Nela se observa que os tratamentos realizados durante o cultivo de $C$. officinalis e os sistemas solventes utilizados nas extrações não influenciaram qualitativamente a composição química dos extratos em relação aos grupos de substâncias pesquisados. No que se refere aos solventes utilizados nas extrações, a princípio, quando se compara a polaridade deles, observa-se que em ambos há uma mistura de um álcool com água e que, pelas proporções, eles terão polaridades assemelhadas. Deste modo, é previsível que os componentes químicos extraídos das drogas vegetais sejam do mesmo tipo.

TABELA 1. RESULTADOS DO SCREENING FITOQUÍMICO DOS EXTRATOS DE C. officinalis

\begin{tabular}{|c|c|c|c|c|c|c|c|c|c|c|c|c|}
\hline \multirow[b]{2}{*}{ Testes $\mathrm{p} /$} & \multicolumn{6}{|c|}{ Extrato hidroetanólico } & \multicolumn{6}{|c|}{ Extrato hidroglicólico } \\
\hline & T1 & T2 & T3 & T4 & T5 & T6 & T1 & T2 & T3 & T4 & T5 & T6 \\
\hline $\begin{array}{l}\text { Fenóis (Taninos } \\
\text { catequínicos) }\end{array}$ & + & + & + & + & + & + & + & + & + & + & + & + \\
\hline Flavonoides & + & + & + & + & + & + & + & + & + & + & + & + \\
\hline Alcaloides & + & + & + & + & + & + & + & + & + & + & + & + \\
\hline Cumarinas & + & + & + & + & + & + & + & + & + & + & + & + \\
\hline Saponinas & + & + & + & + & + & + & + & + & + & + & + & + \\
\hline $\begin{array}{l}\text { Esteroides e } \\
\text { Triterpenos }\end{array}$ & - & - & - & - & - & - & - & - & - & - & - & - \\
\hline $\begin{array}{l}\text { Heterosídeos } \\
\text { Cardiotônicos }\end{array}$ & - & - & - & - & - & - & - & - & - & - & - & - \\
\hline Carotenoides & - & - & - & - & - & - & - & - & - & - & - & - \\
\hline Antraquinonas & - & - & - & - & - & - & - & - & - & - & - & - \\
\hline
\end{tabular}

T1: adubação química; T2: adubação química + cobertura morta de grama seca;

T3: adubação orgânica; T4: adubação orgânica + cobertura morta de grama seca;

T5: sem adubação química ou orgânica + cobertura morta de grama seca;

T6: sem adubação química ou orgânica e sem cobertura morta. 
Os taninos, princípios ativos de estrutura polifenólica, reagem com várias substâncias, produzindo substâncias de coloração diferente ou mesmo se insolubilizando no meio reacional. Nos testes realizados, todos os extratos (hidroetanólicos e hidroglicólicos) produziram com cloreto férrico, alteração de coloração e precipitações, indicando a presença dessa categoria. A cor predominante formada foi esverdeada, concluindo que, possivelmente os taninos presentes sejam os catequínicos. Nunes et al. (2009) também pesquisaram tinturas dessa planta com esse tipo de reativo e reportaram que os resultados são indicativos para presença de fenóis. Sindhu (2010) também evidenciou taninos nos extratos aquosos das folhas dessa planta, porém Kumar et al. (2010) não conseguiu evidenciá-los usando o mesmo tipo de extrato. Khalid e Teixeira da Silva (2012) relatam a não existência de taninos em extratos produzidos com éter de petróleo, metanol, etanol e água.

O teste realizado para flavonoides é mais específico para evidenciar alguns tipos, tais como flavonóis, flavanonas, flavanonóis e xantonas. Todos os extratos testados (hidroetanólicos e hidroglicólicos) mostraram, com maior ou menor facilidade de visualização, a coloração rósea, caracterizando a presença desse tipo de princípio ativo. Nunes et al. (2009), Sandhu (2010), Kumar et al. (2010) e Khalid e Teixeira da Silva (2012) também evidenciaram essa categoria nas amostras testadas. É normal a presença deles em $C$. officinalis, pois na literatura técnica, os marcadores escolhidos para essa espécie são substâncias dessa categoria (FARMACOPEIA BRASILEIRA, 2010).

A presença das saponinas foi observada nos extratos hidroetanólicos e hidroglicólicos tendo como fundamento a característica de produzem espuma quando agitadas (propriedade afrogênica). No entanto, esse resultado é controverso àquele apresentado por Nunes et al. (2009) que, usando o mesmo teste qualitativo, não encontraram saponinas nas tinturas dessa espécie. Por outro lado, Sindhu (2010), através de análise por cromatografia em camada delgada e Kumar et al. (2010), usando abordagem fitoquímica, observaram a presença de saponinas nos extratos aquosos e etanólicos das folhas de C. officinalis. Khalid e Teixeira da Silva (2012) relatam a presença delas em extratos metanólicos, etanólicos e aquosos. Os resultados apresentados no presente trabalho parecem ser conflitantes com o teste realizado nestes mesmos extratos para evidenciação de compostos esteroides e triterpênicos (reação de Liebermann-Burchard). Em todos os extratos, esse teste apresentou resultado negativo. É conhecido que as saponinas podem ter origem triterpênica ou esteroidal (SANTOS, 2007). Deste modo, seria de se esperar que essas análises resultassem em testes positivos. Sandhu (2010) e Kumar et al. (2010) não obtiveram êxito nos testes para evidenciação de esteroides/terpenoides em extratos metanólicos e aquosos das folhas, mas foram evidenciados nos extratos etéreos e clorofórmicos. Khalid e Teixeira da Silva (2012) mencionam a existência de triterpenos em extratos 
produzidos com éter de petróleo e etanol.

Para evidenciação dos alcaloides, nos extratos foi utilizada uma bateria de reativos gerais que envolvem a formação de complexos e precipitação deles no meio reacional (reativos de Dragendorff, Mayer e Bouchardat). Eles foram evidenciados nos extratos hidroetanólicos e hidroglicólicos. Nos trabalhos de Nunes et al. (2009) e Kumar et al. (2010) essa categoria de ativos também foi evidenciada. Sandhu (2010) não pesquisou esse tipo de substância. Por outro lado, Khalid e Teixeira da Silva (2012) informaram que essa categoria de substâncias não foi identificada em extratos produzidos com éter de petróleo, metanol, etanol e água. É importante salientar que, até o momento, nenhum relato em literatura científica do isolamento ou identificação dessa importante categoria de compostos naturais foi realizado em $C$. officinalis.

As cumarinas foram evidenciadas em meio alcalino pelo desenvolvimento de fluorescência sob UV nos extratos hidroetanólicos e extratos hidroglicólicos. Paradoxalmente, Nunes et al. (2009) não as evidenciaram nas tinturas dessa planta. Elas não foram investigadas pelos demais autores, exceto por Khalid e Teixeira da Silva (2012) que mencionam sua existência nessa planta. Há relatos na literatura que hidroxicumarinas já foram isoladas de C. officinalis (MULEY et al., 2009).

Várias categorias de substâncias foram pesquisadas e não evidenciadas em quaisquer dos extratos analisados neste trabalho e por Nunes et al. (2009), como por exemplo heterosídeos cardiotônicos, antraquinonas e carotenoides. Também Kumar et al. (2010) não obtiveram resultados positivos para carotenoides em extratos etanólicos e aquosos das folhas, mas foram evidenciados nos extratos etéreos.

Embora neste trabalho os resultados comparativos do screening fitoquímico sejam conclusivos da semelhança química qualitativa entre os dois tipos de extratos analisados, outras análises desenvolvidas por Borella et al. (2012) com esses mesmos tipos de extratos mostraram que há diferenças quantitativas importantes, principalmente relacionadas com os valores de resíduo seco, não sendo uma alternativa viável a substituição de extratos hidroetanólicos por hidroglicólicos de $C$. officinalis na composição de formulações fitoterápicas.

Ao se analisar os resultados dos índices de espuma das drogas vegetais (tabela 2), observam-se diferenças significativas derivadas do tipo de tratamento realizado durante o cultivo. Em $C$. officinalis, o tipo de cultivo que originou droga com maior índice de espuma foi aquele que utilizou adubação orgânica sem uso de cobertura de solo. Os demais resultados dos tratamentos deste ensaio se mostraram estatisticamente iguais. 
TABELA 2. MÉDIA DO ÍNDICE DE ESPUMA DAS DROGAS VEGETAIS DE C. officinalis

\begin{tabular}{l|c}
\hline \multicolumn{1}{c|}{ Droga vegetal obtida com tratamento: } & Índice de Espuma \\
\hline 1.adubação química & $112 \mathrm{~b}(+/-12,5)$ \\
\hline 2. adubação química + cobertura morta & $108 \mathrm{~b}(+/-14,4)$ \\
\hline 3. adubação orgânica & $198 \mathrm{a}(+/-53,5)$ \\
\hline 4. adubação orgânica + cobertura morta & $181 \mathrm{ab}(+/-32,9)$ \\
\hline $\begin{array}{l}\text { 5. sem adubação química ou orgânica + } \\
\text { cobertura morta }\end{array}$ & $114 \mathrm{~b}(+/-24,8)$ \\
\hline $\begin{array}{l}\text { 6. sem adubação química ou orgânica e sem } \\
\text { cobertura morta }\end{array}$ & $100 \mathrm{~b}(+/-11,3)$ \\
\hline
\end{tabular}

$p<0,05$ - ANOVA seguido pelo teste de comparações múltiplas de Tukey-Kramer. Médias seguidas pelas mesmas letras minúsculas, não diferem estatisticamente entre si. Os números entre parênteses referem-se ao desvio padrão.

Esses resultados vão ao encontro daqueles apresentados por Khalid e Teixeira da Silva (2012) que apontam o cultivo com uso de adubação orgânica em sol pleno como preferencial para obtenção de inflorescências de $C$. officinalis com maior concentração de uma série de compostos fenólicos (como por exemplo, cumarinas, ácidos clorogênicos, entre outros) em detrimento do cultivo com adubação química.

\section{CONCLUSÕES}

Entre os extratos de $C$. officinalis analisados não houve diferenças qualitativas na composição química evidenciando que as condições de cultivo e os sistemas solventes utilizados no processo extrativo não influenciaram esse quesito. Tanto nos extratos hidroetanólicos (tinturas) como nos extratos hidroglicólicos (extrato glicólicos) foram evidenciados os fenóis (taninos catequínicos), flavonoides, saponinas, alcaloides e cumarinas. Não foram evidenciados heterosídeos carditônicos, esteroides/triterpenos, carotenoides e antraquinonas. Nota-se que há grandes divergências entre os resultados dos diversos trabalhos publicados com essa espécie em relação à prospecção fitoquímica.

Com relação às drogas vegetais de $C$. officinalis, houve variabilidade estatística nos seus índices de espuma. Nesse caso, os tratamentos utilizados durante o cultivo foram decisivos para esse resultado. A droga vegetal que exibiu maiores valores do IE foi obtida utilizando adubação orgânica sem utilização de cobertura morta. Deste modo, pode-se indicar esse tipo de tratamento durante o cultivo dessa espécie se o objetivo for produzir droga vegetal derivada das inflorescências de $C$. officinalis mais ricas em 
saponinas espumídicas.

\section{AGRADECIMENTOS}

Agradecemos à Fundação de Amparo à Pesquisa do Estado de São Paulo FAPESP (Processo 2006/64408-3) e ao Conselho Nacional de Desenvolvimento Científico e Tecnológico - CNPq pelo apoio financeiro e bolsas de estudos que nos concederam para a realização deste projeto.

\section{REFERÊNCIAS}

BARBOSA, W.L.R.; QUIGNARD, E.; TAVARES, I.C.C.; PINTO, L.N.; OLIVEIRA, F.Q.; OLIVEIRA, R.M. Manual para análise fitoquímica e cromatográfica de extratos vegetais. Revista Científica da Universidade Federal do Pará. 4:1-19, 2004.

BORELLA, J.C.; CARVALHO, D.M.A. Avaliação comparativa da qualidade de extratos de Calendula officinalis L. (Asteraceae) comercializados em farmácias de manipulação em Ribeirão Preto - SP. Revista Brasileira de Farmácia. 92(1): 11-16, 2011.

BORELLA, J.C.; RIBEIRO, N.S.; TEIXEIRA, J.C.L.; CARVALHO, D.M.A. Avaliação da espalhabilidade e do teor de flavonoides em forma farmacêutica semissólida contendo extratos de Calendula officinalis L. (Asteraceae). Revista de Ciências Farmacêuticas Básica e Aplicada. 31:193-197, 2010.

BORELLA, J.C.; RIBEIRO, N.S.; TEIXEIRA, J.C.L.; CARVALHO, D.M.A. Influência do processo extrativo nas propriedades físico-químicas dos extratos de Calendula officinalis L. (Asteraceae). Revista Eletrônica de Farmácia. 9(2): 25-36, 2012.

BORELLA, J.C.; TEIXEIRA, J.C.L. Avaliação comparativa de certificados de análises de empresas que comercializam tintura de Calendula officinalis L. (Asteraceae). Visão Acadêmica. 14(3), 26-35, 2013.

CITADINI-ZANETTE, V.; NEGRELLE, R.R.B.; BORBA, E.T. Calendula officinalis L. (Asteraceae): aspectos botânicos,ecológicos e usos. Visão Acadêmica. 13(1), 6-23, 2012.

CRAGG, G.M.; NEWMAN, D.J. Biodiversidade: um componente essencial na descoberta de fármacos. In: YUNES, R.A.; CECHINEL FILHO, V. (Orgs.). Química de produtos naturais, novos fármacos e a moderna farmacognosia. Itajaí: Univali, 2009. cap. III, p. 52-81. 
FARMACOPEIA BRASILEIRA. 5a ed. Volume 1 e 2. Agência Nacional de Vigilância Sanitária [internet] 2010. Disponível em: < http://www.anvisa.gov.br/hotsite/cd_ farmacopeia/index.htm >. Acesso em janeiro 2014.

FERNANDES, E.F.A.; MELONI, F.; BORELLA, J.C.; LOPES N. P. Effect of fertilisation and harvest period on polar metabolites of Calendula oficcinalis. Rev. Bras. Farmacogn. 23: 731-735, 2013.

FORMULÁRIO DE FITOTERÁPICOS DA FARMACOPEIA BRASILEIRA. 1ª ed. Agência Nacional de Vigilância Sanitária [internet] 2010. Disponível em: <http://www.anvisa.gov.br/farmacopeiabrasileira/conteudo/Formulario_de_Fitoterapic os_da_Farmacopeia_Brasileira.pdf.>.Acesso em janeiro 2014.

GOBBO-NETO, L.; LOPES, N.P. Plantas medicinais: fatores de influência no conteúdo de metabólitos secundários. Quim. Nova. 30(2): 374-381, 2007.

GOTTLIEB, O.R.; BORIN, M.R.M.B. Químico-biologia quantitativa: um novo paradigma? Quim. Nova. 35(11): 2105-2114, 2012.

KHALID, K.A.; TEIXEIRA DA SILVA, J.A. Biology of Calendula officinalis Linn.: focus on pharmacology, biological activities and agronomic practices. Medicinal and Aromatic Plant Science and Biotechnology. 6(1): 12-27, 2012.

KUMAR, N.; SHARMA, J.; SHARMA, S. Pharmacognostical and phytochemical investigation of Calendula officinalis. Journal of Advanced Scientific Research. 1(1): 61-66, 2010.

LEACH, M.J. Calendula officinalis and wound healing: a systematic review. Wounds. 20(8): 1-7, 2008.

MULEY, B.P.; KHADABADI, S.S.; BANARASE, N.B. Phytochemical constituents and pharmacological activities of Calendula officinalis Linn (Asteraceae): a review. Tropical Journal of Pharmaceutical Research. 8(5): 455-465, 2009.

NUNES, K.M.; BARBOSA, W.L.R.; OZELA, E.F.; SILVA JÚNIOR, J.O.C. Padronização da tintura de Calendula officinalis $L$. para seu emprego em formulações semi-solidas fitoterápicas. Lat. Am. J. Pharm. 28 (3): 344-350, 2009.

SANTOS, R.I. Metabolismo básico e origem dos metabólitos secundários. In: SIMÕES, 
C.M.O.; SCHENKEL, E.P.; GOSMANN, G.; MELLO, J.C.P.; MENTZ, L.A.; PETROVICK P.R. (Orgs.). Farmacognosia - da planta ao medicamento. $6^{a}$ ed. Porto Alegre: Editora da UFRGS; Florianópolis: Editora da UFSC, 2007. cap. 16, p.403-434.

SINDHU, C.G. Phytochemical screening of Calendula officinalis Linn. leaf extract by TLC. International Journal of Research in Ayurveda \& Pharmacy. 1 (1):131-134, 2010.

SONAGLIO, D.; ORTEGA, G.G.; PETROVIK, P.R.; BASSANI, V.L. Desenvolvimento tecnológico e produção de fitoterápicos. In: SIMÕES, C.M.O.; SCHENKEL, E.P.; GOSMANN, G.; MELLO, J.C.P.; MENTZ, L.A.; PETROVICK P.R. (Orgs.). Farmacognosia - da planta ao medicamento. $6^{a}$ ed. Porto Alegre: Editora da UFRGS; Florianópolis: Editora da UFSC, 2007. cap. 13, p.289-326. 